\title{
Mismatch repair single nucleotide polymorphisms and thyroid cancer susceptibility
}

\author{
LUÍS S. SANTOS ${ }^{1,2}$, SUSANA N. SILVA ${ }^{1}$, OCTÁVIA M. GIL ${ }^{1,3}$, \\ TERESA C. FERREIRA ${ }^{4}$, EDWARD LIMBERT ${ }^{4}$ and JOSÉ RUEFF ${ }^{1}$
}

\begin{abstract}
${ }^{1}$ Centre for Toxicogenomics and Human Health (ToxOmics), Genetics, Oncology and Human Toxicology, NOVA Medical School, Faculdade de Ciências Médicas, Universidade Nova de Lisboa, 1169-056 Lisboa; ${ }^{2}$ Centre for Interdisciplinary Research in Health (CIIS), Health Sciences Institute (ICS), Universidade Católica Portuguesa, 3504-505 Viseu; ${ }^{3}$ Center for Nuclear Sciences and Technologies $\left(C^{2} \mathrm{TN}\right)$, Instituto Superior Técnico, Universidade de Lisboa, 2695-066 Bobadela LRS; ${ }^{4}$ Department of Nuclear Medicine, Instituto Português de Oncologia de Lisboa,
\end{abstract} 1099-023 Lisboa, Portugal

Received September 13, 2017; Accepted December 14, 2017

DOI: $10.3892 / \mathrm{ol} .2018 .8103$

\begin{abstract}
Thyroid cancer (TC) is the most common endocrine malignancy and its incidence continues to rise worldwide. Ionizing radiation exposure is the best established etiological factor. Heritability is high; however, despite valuable contribution from recent genome-wide association studies, the current understanding of genetic susceptibility to TC remains limited. Several studies suggest that altered function or expression of the DNA mismatch repair (MMR) system may contribute to TC pathogenesis. Therefore, the present study aimed to evaluate the potential role of a panel of MMR single nucleotide polymorphisms (SNPs) on the individual susceptibility to well-differentiated TC (DTC). A case-control study was performed involving 106 DTC patients and 212 age- and gender-matched controls, who were all Caucasian Portuguese. Six SNPs present in distinct MMR genes (MLH1 rs1799977, MSH3 rs26279, MSH4 rs5745325, PMS1 rs5742933, MLH3 rs175080 and MSH6 rs1042821) were genotyped through TaqMan $^{\circledR}$ assays and genotype-associated risk estimates were calculated. An increased risk was observed in MSH6 rs1042821 variant homozygotes [adjusted odds ratio $(\mathrm{OR})=3.42,95 \% \mathrm{CI}$ : 1.04-11.24, $\mathrm{P}=0.04$, under the co-dominant model; adjusted $\mathrm{OR}=3.84,95 \% \mathrm{CI}: 1.18-12.44, \mathrm{P}=0.03$, under the recessive model]. The association was especially evident for the follicular histotype and female sex. The association was also
\end{abstract}

Correspondence to: Professor José Rueff, Centre for Toxicogenomics and Human Health (ToxOmics), Genetics, Oncology and Human Toxicology, NOVA Medical School, Faculdade de Ciências Médicas, Universidade Nova de Lisboa, Campo dos Mártires da Pátria, 130, 1169-056 Lisboa, Portugal

E-mail: jose.rueff@nms.unl.pt

Key words: MSH6, DNA repair, mismatch repair, single nucleotide polymorphism, genetic susceptibility, thyroid cancer apparent when MSH6 was analysed in combination with other MMR SNPs such as MSH3 rs26279. Interestingly, two other SNP combinations, both containing the MSH6 heterozygous genotype, were associated with a risk reduction, suggesting a protective effect for these genotype combinations. These data support the idea that MMR SNPs such as MSH6 rs1042821, alone or in combination, may contribute to DTC susceptibility. This is coherent with the limited evidence available. Nevertheless, further studies are needed to validate these findings and to establish the usefulness of these SNPs as genetic susceptibility biomarkers for DTC so that, in the near future, cancer prevention policies may be optimized under a personalized medicine perspective.

\section{Introduction}

Despite accounting for only $2 \%$ of all human cancers, thyroid cancer (TC) is the most common endocrine malignancy. Its incidence continues to rise worldwide, being one of the cancers with the highest incidence among adolescent and young adults (ages 15-39 years) and three times more frequent in women than in men $(1,2)$. TC is usually classified with respect to histological and clinical criteria: Papillary and follicular TC, representing $70-80 \%$ and $10-20 \%$ of cases, respectively, are the two most common varieties. Both tend to grow slowly and are often considered together as well-differentiated TC (DTC) $(1,3)$.

DTC is generally accepted as a multifactorial disease (3). Among the several risk factors suggested to contribute to DTC, exposure to ionizing radiation (IR) remains the best-established one $(1,4)$. Heritability is high (familial risk is one of the highest among cancers not showing typical Mendelian inheritance), suggesting that genetic factors (most likely, multiple common low-penetrance or rare moderate-penetrance alleles) strongly contribute to DTC predisposition (5). Much effort has been made to identify such susceptibility variants. The most robust evidence is for markers at 9q22.33 (FOXE1), 14q13.3 (NKX2-1), 2q35 (DIRC3) and 8p12 (NRG1), as variants in 
these regions have been repeatedly associated with DTC through several genome-wide association studies (GWASs), confirmed in follow-up studies and independently replicated across different populations (6-12). Additional markers have recently been suggested $(8,10-13)$ but still require confirmation and replication. Overall, the number of confirmed GWAS-proposed DTC risk alleles is still very limited (14) and, more importantly, explains only a relatively small proportion of the estimated heritability of DTC $(11,15,16)$.

Multiple germ-line single nucleotide polymorphisms (SNPs) within genes involved in critical cellular processes-e.g., DNA repair, cell-cycle control and apoptosis, intracellular signalling, endobiotic or xenobiotic metabolism, thyroid physiology-have also been associated with DTC susceptibility through candidate-gene association studies (CGASs) [reviewed in $(5,17)]$. While most of these findings have not been properly replicated, some could, as recently demonstrated (14), represent true associations with DTC. The identification of additional variants potentially involved in DTC susceptibility may explain part of the missing heritability of the disease and is therefore highly desirable. Considering the important role that DNA-damaging agents such as IR play in DTC aetiology, DNA repair SNPs would be particularly interesting candidates. Many, across the main DNA repair pathways-BER $(18,19)$, NER $(20,21)$, NHEJ $(22,23)$ and HR (24-26)-have already been associated with DTC. To our knowledge, DNA mismatch repair (MMR) SNPs have not yet been investigated.

The MMR pathway plays a crucial role in post-replication repair: It recognizes base-base mispairs and insertion/deletion loops that, in spite of the proofreading function of DNA polymerases, inescapably arise during replication. MMR thus prevents base substitutions or repeat sequence instability, greatly increasing DNA replication fidelity and safeguarding genomic integrity (27). MMR also participates, among other cellular processes (e.g., mitotic and meiotic recombination, immunoglobulin class switching), in the recognition of DNA damage induced by genotoxic chemicals, UV light, IR or oxidative stress (e.g., oxidative lesions, double strand breaks, pyrimidine dimers and inter-strand crosslinks) and subsequent repair (in cooperation with other repair pathways) or damage-induced cytotoxicity (downstream signalling for cell cycle arrest and apoptosis) (28-30). MMR's role is therefore critical to carcinogenesis: loss of MMR (e.g., inactivating mutation) greatly increases the rate of spontaneous mutation, leading to a mutator phenotype, and results in microsatellite instability (MSI), a hallmark of MMR defects $(27,29,31)$. Not surprisingly, heterozygous germline MMR mutations (e.g., $M L H 1, M S H 2, M S H 6$ or PMS2) give rise to Lynch syndrome (LS), an autosomal dominant condition (hereditary nonpolyposis colorectal cancer, HNPCC) which strongly predisposes to early-onset colorectal cancer (CRC) and several extracolonic tumours, all typically presenting MSI. MMR mutations and epigenetic silencing (e.g., $M L H 1$ promoter methylation) are also being increasingly implicated in a growing range of tumours $(27,31,32)$.

Interestingly, MMR mutations are increasingly being detected in TC cases $(33,34)$ [mutation frequency correlating with progression from papillary to more aggressive TC phenotypes (35)] and TC, despite not being part of the usual
LS tumour spectrum, has been incidentally observed among LS patients (36-40). The notion that MMR deficiency may contribute to TC pathogenesis and/or progression is biologically plausible since the MMR pathway is involved in the repair and damage response to IR-induced lesions such as 8-oxoGuanine (29). Supplementary evidence (reviewed in (41) further supports this hypothesis: 1) $M L H 1$ promoter methylation occurs in TC and is associated with lymph node metastasis and BRAF mutation; 2) High levels of MSI have been reported in DTC; and 3) altered MLH1, PMS1 and MSH2 expression has been reported in TC.

As such, it is possible that MMR pathway SNPs, through interference with DNA damage response and/or repair capacity in thyroid cells, could contribute to DTC susceptibility. Since this hypothesis has not yet been explored, we undertook a hospital-based case-control study in a Caucasian Portuguese population, to evaluate the potential modifying role of a panel of SNPs in MMR genes on the individual susceptibility to non-familial DTC. Identifying SNPs which may serve as DTC susceptibility biomarkers may contribute to the identification of individuals who are at increased risk for DTC and, eventually, the optimization of cancer prevention procedures.

\section{Materials and methods}

Ethical statement. This study was approved by the local ethics committees of the involved institutions and carried out in compliance with the Helsinki Declaration. At recruitment, written informed consent was obtained from each study subject and anonymity was guaranteed.

Study subjects. A total of 318 participants, all of which Caucasian Portuguese, were enrolled in this study: 106 histologically confirmed DTC patients subject to Iodine-131 treatment in the Department of Nuclear Medicine of the Portuguese Oncology Institute, Lisbon, Portugal and 212 age ( \pm 2 years) and gender-matched controls (two for each case), selected from unrelated subjects who were seeking healthcare for non-neoplasic pathology at São Francisco Xavier Hospital, Lisbon, Portugal. For controls, age at diagnosis was defined as the matched case age of diagnosis. The recruitment of both patients and controls was based on previously described (21) inclusion and exclusion criteria. At recruitment, a standard questionnaire was administered through face-to-face interviews by trained interviewers to obtain information on demographic characteristics (e.g., gender, age, occupation), family history of cancer, lifestyle habits (e.g., smoking, alcohol drinking) and IR exposure. According to the information collected, none of the study participants had been previously exposed to relevant (i.e. other than that from natural and standard diagnostic sources) levels of ionizing radiation (from therapeutic or occupational sources, e.g. none of the study participants worked or lived nearby a nuclear power plant). Detailed clinical and pathological investigation was also performed. For the purpose of smoking status, former smokers who gave up smoking either 2 years before DTC diagnosis or 2 years before their inclusion as controls were considered as non-smokers. The participation rate was 95\% and blood samples were available for all subjects. 
Table I. Selected SNPs and detailed information on the corresponding base and amino acid exchanges, MAF and TaqMan ${ }^{\circledR}$ assay used for genotyping.

\begin{tabular}{|c|c|c|c|c|c|c|}
\hline Gene & Location & db SNP ID (rs no.) ${ }^{\mathrm{a}}$ & Base change & Aminoacid change & $\operatorname{MAF}(\%)^{\mathrm{a}}$ & TaqMan $^{\circledR}$ assay \\
\hline$M L H 1$ & $3 \mathrm{p} 22.2$ & rs1799977 & $\mathrm{A} \rightarrow \mathrm{G}$ & Ile219Val & 13.0 & C__1219076_20 \\
\hline MSH3 & $5 q 14.1$ & rs26279 & $\mathrm{A} \rightarrow \mathrm{G}$ & Thr1045Ala & 28.0 & C__800002_1_ \\
\hline MSH4 & $1 \mathrm{p} 31.1$ & rs5745325 & $\mathrm{G} \rightarrow \mathrm{A}$ & Ala97Thr & 21.3 & C__3286081_10 \\
\hline PMS1 & $2 q 32.2$ & rs5742933 & $\mathrm{G} \rightarrow \mathrm{C}$ & $-{ }^{b}$ & 21.9 & C__29329633_10 \\
\hline$M L H 3$ & $14 q 24.3$ & rs175080 & $\mathrm{G} \rightarrow \mathrm{A}$ & Pro844Leu & 36.4 & C__1082805_10 \\
\hline MSH6 & $2 \mathrm{p} 16.3$ & rs1042821 & $\mathrm{C} \rightarrow \mathrm{T}$ & Gly39Glu & 20.1 & C__8760558_10 \\
\hline
\end{tabular}

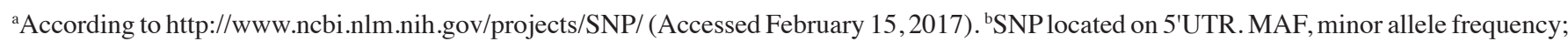
SNP, single nucleotide polymorphism.

SNP selection. Using the publicly available NCBI SNP database (http://www.ncbi.nlm.nih.gov/snp/, accessed February 15, 2017), a comprehensive set of potentially functional SNPs covering the MMR pathway were selected for genotyping. In order to be eligible, SNPs had to i) alter the amino acid sequence (missense SNPs); ii) exhibit minor allele frequency (MAF) greater than 0.10 ; and iii) have been previously referred to in MEDLINE (https://www.ncbi.nlm.nih.gov/pubmed/, accessed February 15, 2017). A total of five SNPs, specifically, rs1799977 (MLH1), rs26279 (MSH3), rs5745325 (MSH4), rs175080 (MLH3) and rs1042821 (MSH6), fulfilled these criteria and were thus analysed. In addition, since no PMS1 SNP fulfilled all of these criteria, rs5742933- a common (MAF >0.10) 5'UTR SNP which is located within the PMS1 promoter region (potentially regulatory role on transcription) and is the most frequently quoted PMS1 SNP- was also included in the study. Table I summarizes the genomic location, base and amino acid exchange and MAF of the selected SNPs.

DNA extraction and genotyping. After informed consent, peripheral venous blood samples from each study subject were collected into $10 \mathrm{ml}$ heparinised tubes and stored at $-80^{\circ} \mathrm{C}$. Genomic DNA was extracted from these samples by using the commercially available QIAamp ${ }^{\circledR}$ DNA mini kit (Qiagen $\mathrm{GmbH}$, Hilden, Germany) according to the manufacturer's protocols. DNA extracts were kept at $-20^{\circ} \mathrm{C}$ until analysis.

In order to assure uniformity in DNA content $(2.5 \mathrm{ng} / \mu \mathrm{l})$ prior to genotyping, DNA quantity was assessed fluorimetrically in all samples using the Quant-iT ${ }^{\mathrm{TM}}$ Picogreen $^{\circledR}$ dsDNA Assay kit (Invitrogen; Thermo Fisher Scientific, Inc., Waltham, MA, USA) and a Zenyth 3100 plate reader (Anthos Labtec Instruments, Salzburg, Austria).

SNP genotyping was carried out using the Taqman ${ }^{\circledR}$ allelic discrimination assay on a 96-well ABI 7300 Real Time PCR system (Applied Biosystems; Thermo Fisher Scientific, Inc.), following the manufacturer's instructions. Commercial pre-designed assay primers and probes, purchased from Applied Biosystems; Thermo Fisher Scientific, Inc., were used for every SNP and are listed in Table I. The amplification conditions consisted of an initial activation step (10 min, $95^{\circ} \mathrm{C}$ ), followed by $\geq 40$ amplification cycles of denaturation $\left(15 \mathrm{sec}, 92^{\circ} \mathrm{C}\right)$ and annealing/extension $\left(60 \mathrm{sec}, 60^{\circ} \mathrm{C}\right)$. The fluorescence intensity emitted by VIC and FAM dyes in each well was detected (60 sec) and analysed with Applied Biosystems sequence detection software (System SDS version 1.3.1).

To assure accuracy of the genotyping and avoid variant misclassification, four negative controls (wells containing no DNA) were included in each plate. Genotyping of inconclusive samples was repeated. Also, for quality control, $10-15 \%$ of the samples were randomly selected and run in duplicates. $100 \%$ concordance between experiments was observed.

Statistical analysis. Prior to analysis, an exact probability test available in SNPStats software (42) was used to check whether genotype distributions for each studied SNP deviated significantly from Hardy-Weinberg equilibrium (HWE).

Since all variables considered were categorical or categorized (e.g., age), descriptive statistics were presented as frequencies and percentages.

The distribution of demographic variables such as gender, age group and smoking status and of genotype frequencies was compared between groups through Chi-square or two-sided Fisher's exact test for $2 \times 2$ or $2 \times 3$ contingency tables, respectively.

For all elected SNPs, genotype-associated risk of DTC was estimated by binary logistic regression analysis and expressed as both crude and adjusted odds ratios (OR) and $95 \%$ confidence intervals (CI). Risk estimates were calculated under codominant, dominant, recessive and log-additive genetic models. Adjustment, when performed, included terms for gender (male/female), age group ( $<30,30-49,50-69$ and $\geq 70$ years) and smoking habits (smokers/non-smokers). The most common homozygous genotype, female gender, lower age group and non-smoking status were taken as reference for the purpose of such calculations. The remaining information that was collected on demographic characteristics (e.g. occupation), family history of cancer, lifestyle habits (e.g. alcohol drinking) and prior IR exposure was not suitable for rigorous quantitative transformation and, therefore, not included in the adjustment.

Stratified analysis according to histological type of tumour (papillary or follicular TC), gender and age was also performed. Additionally, we conducted a genotype interaction analysis (combination of alleles) in order to investigate the 
combined effect of different pairs of SNPs on DTC risk. All possible combinations were analysed. For each pair of SNPs, the combination of the most common homozygous genotypes of each individual SNP was taken as the reference category. Paired genotypes with frequency $<5 \%$ in the control group were pooled together.

Finally, the chromosomic location of the variants included in this study was compared to that of DTC markers previously reported in GWAS. Linkage disequilibrium between co-localized variants in European populations was verified in silico through the use of LDLink (43), a publicly available web-based application that uses Phase 3 haplotype data from the 1,000 Genomes Project to calculate pairwise LD between user-input variants in different population groups.

This was a 'proof of concept' study to ascertain whether MMR variants might be linked to DTC. Bonferroni adjustment was not used because it is too conservative. Also, the complement of the false negative rate $\beta$ to compute the power of a test (1- $\beta$ ) was not taken into account at this stage since further studies with more patients and controls should be undertaken to change over this preliminary study into a confirmatory positive one.

The statistical analysis was done with SPSS 22.0 (IBM SPSS Statistics for Windows, version 22.0; IBM Corp, Armonk, NY, USA) except for HWE deviation assessment, MAF calculations, haplotype estimation and linkage disequilibrium (LD) analysis which were performed using the SNPstats Software (42). Two-tailed $\mathrm{P}<0.05$ was considered to indicate a statistically significant difference.

\section{Results}

The demographic characteristics of the 106 DTC cases and their 212 age and gender-matched controls are depicted in Table II. The mean age for each group was 52 years (range 19-77 in the patient group and 18-77 in the control group). Female patients significantly outnumbered male patients, in accordance with the worldwide estimation for gender distribution in DTC $(1,2)$. A total of $11.3 \%$ of patients were categorized as smokers. No statistically significant difference between groups was observed concerning age distribution, gender and smoking habits. Regarding DTC histological classification, 78 (73.6\%) patients were diagnosed as of the papillary type, while $28(26.4 \%)$ were diagnosed as of the follicular type. All cancer patients were incident cases and none of the controls had a family history of cancer.

Table III summarizes the results for MAF, genotypic frequencies and crude/adjusted ORs of the six MMR pathway SNPs selected in our study. The genotype distributions of the studied SNPs were in HWE $(\mathrm{P} \geq 0.05)$, in both case and control groups. No relevant LD was observed between the studied SNPs (data not shown). When comparing, for each of the studied SNPs, the genotype frequency distribution between cases and controls, a significant difference was observed only for MSH6 rs1042821 ( $\mathrm{P}=0.04$, on the codominant and recessive models). Statistical significance was not attained when assuming a dominant model of inheritance $(\mathrm{P}=0.54)$. No additional significant differences were found, irrespective of the model of inheritance assumed. When performing logistic regression analysis, a significant DTC risk increase
Table II. General characteristics for the DTC case $(\mathrm{n}=106)$ and control $(n=212)$ populations.

\begin{tabular}{lrcc}
\hline Characteristics & Controls n(\%) & Cases n (\%) & P-value $^{\mathrm{c}}$ \\
\hline $\begin{array}{l}\text { Gender } \\
\text { Male }\end{array}$ & $31(14.6)$ & $16(15.1)$ & 1.00 \\
Female & $181(85.4)$ & $90(84.9)$ & \\
Age $^{\mathrm{a}, \mathrm{b}}$ & & & \\
$<30$ & $10(4.7)$ & $4(3.8)$ & 0.98 \\
$30-49$ & $75(35.4)$ & $38(35.8)$ & \\
$50-69$ & $98(46.2)$ & $49(46.2)$ & \\
$\geq 70$ & $29(13.7)$ & $15(14.2)$ & \\
Smoking habits & & & \\
Non-smokers & $174(82.1)$ & $94(88.7)$ & 0.19 \\
Smokers & $36(17.0)$ & $12(11.3)$ & \\
Missing & $2(0.9)$ & $0(0.0)$ & \\
\hline
\end{tabular}

${ }^{\mathrm{a}} \mathrm{Age}$ of diagnosis, for cases. ${ }^{\mathrm{b}} \mathrm{Age}$ at the time of diagnosis of the matched case, for controls. 'P-value for cases vs. control group determined by two-sided Fisher's exact test (gender, smoking habits) or $\chi^{2}$ test (age). DTC, well-differentiated thyroid cancer.

was observed in MSH6 rs1042821 variant allele homozygotes, after adjustment for age, gender and smoking status $(\mathrm{Glu} / \mathrm{Glu}$ vs. Gly/Gly: adjusted OR=3.42, 95\% CI: $1.04-11.24, \mathrm{P}=0.04$; Glu/Glu vs. Gly/Gly+Gly/Glu: adjusted OR=3.84, 95\% CI: 1.18-12.44, $\mathrm{P}=0.03)$. This association was also apparent without covariate adjustment when assuming a recessive model of inheritance (Glu/Glu vs. Gly/Gly+Gly/Glu: OR=3.35, 95\% CI: 1.07-10.50, $\mathrm{P}=0.04)$. No significant associations with DTC risk were observed for the remaining SNPs analysed in this study, irrespective of the model assumed.

Since DTC comprises two distinct histological types (papillary and follicular), affects women more than men and is the most incident malignancy in the 15-39 years age group $(1,2)$, patients and controls were stratified on the basis of these criteria, i.e., histological tumour type, gender and age, in order to identify any subgroup-specific risk association. As shown in Table IV, stratification of subjects according to histological criteria showed that the association between the homozygous variant genotype of MSH6 rs1042821 and DTC risk, observed in the complete set of patients, was also present in the follicular subset (adjusted OR $=20.98,95 \%$ CI: 1.08-406.53, $\mathrm{P}=0.04$, under the co-dominant model; adjusted $\mathrm{OR}=23.70,95 \%$ CI: $1.25-449.32, \mathrm{P}=0.04$, under the recessive model) but absent from the papillary subset, suggesting a histological type-specific SNP effect. Also in the follicular subset, a significant difference in the genotype frequency distribution of $M L H 3$ rs 175080 was observed $(\mathrm{P}=0.04$, in the dominant model, data not shown). On binary logistic regression analysis, significantly increased follicular TC risk was observed in MLH3 rs175080 variant allele carriers (OR=3.95, 95\% CI: 1.05-14.81, $\mathrm{P}=0.04)$. After gender stratification (Table IV), the frequency distribution of MSH6 rs1042821 genotypes differed significantly between female DTC patients and their age and gender-matched controls $(\mathrm{P}=0.02$, in the codominant model, 
Table III. Genotype distribution in case $(n=106)$ and control $(n=212)$ populations and associated DTC risk (crude and adjusted ORs).

\begin{tabular}{|c|c|c|c|c|c|c|c|}
\hline \multirow[b]{2}{*}{ Genotype } & \multicolumn{2}{|c|}{ MAF } & \multicolumn{2}{|c|}{ Genotype frequency } & \multirow[b]{2}{*}{ P-value ${ }^{a}$} & \multirow[b]{2}{*}{ OR $(95 \% \mathrm{CI})$} & \multirow[b]{2}{*}{ Adjusted OR (95\% CI) } \\
\hline & Controls & Cases & Controls n $(\%)$ & Cases n $(\%)$ & & & \\
\hline MLH1 rs 1799977 & & & $212(100)$ & $105(100)$ & & & \\
\hline Ile/Ile & G: 0.34 & G: 0.30 & $93(43.9)$ & $48(45.7)$ & 0.42 & 1 (Reference) $^{\mathrm{d}}$ & 1 (Reference) $^{\mathrm{d}}$ \\
\hline Ile/Val & & & $95(44.8)$ & $50(47.6)$ & & $1.02(0.63-1.66)$ & $1.02(0.62-1.68)$ \\
\hline $\mathrm{Val} / \mathrm{Val}$ & & & $24(11.3)$ & $7(6.7)$ & & $0.57(0.23-1.41)$ & $0.56(0.22-1.40)$ \\
\hline Dominant model & & & $119(56.1)$ & $57(54.3)$ & 0.81 & $0.93(0.58-1.49)$ & $0.93(0.58-1.50)$ \\
\hline Recessive model & & & $24(11.3)$ & $7(6.7)$ & 0.23 & $0.56(0.23-1.34)$ & $0.55(0.23-1.34)$ \\
\hline Log-additive model & & & - & - & - & $0.86(0.59-1.23)$ & $0.85(0.59-1.24)$ \\
\hline MSH3 rs 26279 & & & $211(100)$ & $105(100)$ & & & \\
\hline $\mathrm{Thr} / \mathrm{Thr}$ & G: 0.35 & G: 0.33 & $93(44.1)$ & $48(45.7)$ & 0.89 & 1 (Reference) $^{\mathrm{d}}$ & 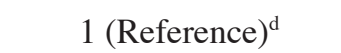 \\
\hline Thr/Ala & & & $90(42.7)$ & 45 (42.9) & & $0.97(0.59-1.60)$ & $0.94(0.57-1.56)$ \\
\hline Ala/Ala & & & $28(13.3)$ & $12(11.4)$ & & $0.83(0.39-1.78)$ & $0.80(0.37-1.72)$ \\
\hline Dominant model & & & $118(55.9)$ & $57(54.3)$ & 0.81 & $0.94(0.59-1.50)$ & $0.91(0.56-1.46)$ \\
\hline Recessive model & & & $28(13.3)$ & $12(11.4)$ & 0.72 & $0.84(0.41-1.73)$ & $0.82(0.40-1.70)$ \\
\hline Log-additive model & & & - & - & - & $0.93(0.66-1.31)$ & $0.91(0.64-1.28)$ \\
\hline MSH4 rs5745325 & & & $212(100)$ & $106(100)$ & & & \\
\hline Ala/Ala & A: 0.33 & A: 0.27 & $97(45.8)$ & $57(53.8)$ & 0.38 & 1 (Reference) $^{\mathrm{d}}$ & 1 (Reference) $^{\mathrm{d}}$ \\
\hline Ala/Thr & & & $91(42.9)$ & $40(37.7)$ & & $0.75(0.46-1.23)$ & $0.75(0.45-1.23)$ \\
\hline $\mathrm{Thr} / \mathrm{Thr}$ & & & $24(11.3)$ & $9(8.5)$ & & $0.64(0.28-1.47)$ & $0.64(0.28-1.48)$ \\
\hline Dominant model & & & $115(54.2)$ & $49(46.2)$ & 0.19 & $0.73(0.45-1.16)$ & $0.72(0.45-1.16)$ \\
\hline Recessive model & & & $24(11.3)$ & $9(8.5)$ & 0.56 & $0.73(0.33-1.63)$ & $0.72(0.32-1.63)$ \\
\hline Log-additive model & & & - & - & - & $0.78(0.54-1.11)$ & $0.78(0.54-1.11)$ \\
\hline PMS1 rs5742933 & & & $212(100)$ & $104(100)$ & & & \\
\hline $\mathrm{G} / \mathrm{G}$ & C: 0.18 & C: 0.17 & $144(67.9)$ & $73(70.2)$ & 0.90 & 1 (Reference) $^{\mathrm{d}}$ & 1 (Reference) $^{\mathrm{d}}$ \\
\hline $\mathrm{G} / \mathrm{C}$ & & & $58(27.4)$ & $27(26.0)$ & & $0.92(0.54-1.57)$ & $0.88(0.51-1.51)$ \\
\hline $\mathrm{C} / \mathrm{C}$ & & & $10(4.7)$ & $4(3.8)$ & & $0.79(0.24-2.60)$ & $0.76(0.23-2.60)$ \\
\hline Dominant model & & & $68(32.1)$ & $31(29.8)$ & 0.70 & $0.90(0.54-1.50)$ & $0.86(0.51-1.45)$ \\
\hline Recessive model & & & $10(4.7)$ & $4(3.8)$ & 1.00 & $0.81(0.25-2.64)$ & $0.80(0.24-2.67)$ \\
\hline Log-additive model & & & - & - & - & $0.90(0.59-1.38)$ & $0.88(0.57-1.35)$ \\
\hline MLH3 rs 175080 & & & $212(100)$ & $106(100)$ & & & \\
\hline Pro/Pro & A: 0.46 & A: 0.51 & $60(28.3)$ & $22(20.8)$ & 0.34 & 1 (Reference) $^{\mathrm{d}}$ & $1{\text { (Reference })^{\mathrm{d}}}^{-}$ \\
\hline Pro/Leu & & & $109(51.4)$ & $59(55.7)$ & & $1.48(0.83-2.64)$ & $1.50(0.83-2.71)$ \\
\hline Leu/Leu & & & $43(20.3)$ & $25(23.6)$ & & $1.59(0.79-3.17)$ & $1.60(0.79-3.22)$ \\
\hline Dominant model & & & $152(71.7)$ & $84(79.2)$ & 0.17 & $1.51(0.86-2.63)$ & $1.53(0.87-2.69)$ \\
\hline Recessive model & & & $43(20.3)$ & $25(23.6)$ & 0.56 & $1.21(0.69-2.12)$ & $1.21(0.69-2.12)$ \\
\hline Log-additive model & & & - & - & - & $1.26(0.90-1.77)$ & $1.26(0.89-1.78)$ \\
\hline MSH6 rs 1042821 & & & $210(100)$ & $106(100)$ & & & \\
\hline Gly/Gly & $\mathrm{T}: 0.21$ & $\mathrm{~T}: 0.22$ & $127(60.5)$ & $68(64.2)$ & $0.04^{c}$ & $1{\text { Reference })^{\mathrm{d}}}$ & 1 (Reference) $^{\mathrm{d}}$ \\
\hline Gly/Glu & & & $78(37.1)$ & $30(28.3)$ & & $0.72(0.43-1.20)$ & $0.73(0.43-1.23)$ \\
\hline Glu/Glu & & & $5(2.4)$ & $8(7.5)$ & & $2.99(0.94-9.49)$ & $3.42(1.04-11.24)^{\mathrm{c}}$ \\
\hline Dominant model & & & $83(39.5)$ & $38(35.8)$ & 0.54 & $0.86(0.53-1.39)$ & $0.87(0.54-1.43)$ \\
\hline Recessive model & & & $5(2.4)$ & $8(7.5)$ & $0.04^{c}$ & $3.35(1.07-10.50)^{\mathrm{c}}$ & $3.84(1.18-12.44)^{c}$ \\
\hline Log-additive model & & & - & - & - & $1.05(0.70-1.57)$ & $1.08(0.71-1.63)$ \\
\hline
\end{tabular}

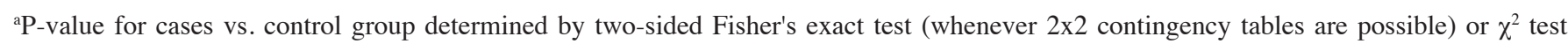
(remaining cases). ${ }^{b}$ ORs were adjusted for gender (male and female), age ( $<30,30-49,50-69, \geq 70$ years) and smoking status (non-smoker and smoker). ${ }^{c}$ Significant results $(\mathrm{P}<0.05)$ highlighted in bold. ${ }^{\mathrm{d}}$ The reference comparator for OR calculations. DTC, well-differentiated thyroid cancer; MAF, minor allele frequency; OR, odds ratio; CI, confidence interval. 
Table IV. Genotype distribution in the case population $(n=106)$ and associated DTC risk (crude and adjusted ORs), after stratification according to histological type, gender and age. ${ }^{\text {a }}$

A, Histological type

\begin{tabular}{|c|c|c|c|c|c|c|}
\hline \multirow[b]{2}{*}{ Genotype } & \multicolumn{3}{|c|}{ Papillary carcinoma } & \multicolumn{3}{|c|}{ Follicular carcinoma } \\
\hline & $\mathrm{n}(\%)$ & OR $(95 \% \mathrm{CI})$ & $\begin{array}{c}\text { Adjusted } \\
\text { OR }(95 \% \mathrm{CI})^{\mathrm{b}}\end{array}$ & $\mathrm{n}(\%)$ & OR $(95 \% \mathrm{CI})$ & $\begin{array}{c}\text { Adjusted } \\
\text { OR }(95 \% \mathrm{CI})^{\mathrm{b}}\end{array}$ \\
\hline MSH6 rs 1042821 & $78(100)$ & & & $28(100)$ & & \\
\hline Gly/Gly & $49(62.8)$ & 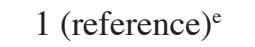 & 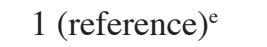 & $19(67.9)$ & 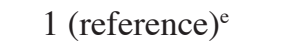 & 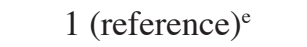 \\
\hline Gly/Glu & $24(30.8)$ & $0.74(0.41-1.32)$ & $0.74(0.41-1.35)$ & $6(21.4)$ & $0.65(0.22-1.91)$ & $0.76(0.24-2.35)$ \\
\hline Glu/Glu & $5(6.4)$ & $2.30(0.59-8.95)$ & $2.47(0.61-9.89)$ & $3(10.7)$ & $5.84(0.57-60.03)$ & $20.98(1.08-406.53)^{\mathrm{c}}$ \\
\hline Dominant model & $29(37.2)$ & $0.83(0.48-1.46)$ & $0.85(0.48-1.49)$ & $9(32.1)$ & $0.92(0.35-2.43)$ & $1.10(0.39-3.07)$ \\
\hline Recessive model & $5(6.4)$ & $2.57(0.67-9.85)$ & $2.74(0.69-10.84)$ & $3(10.7)$ & $6.60(0.65-66.63)$ & $23.70(1.25-449.32)^{\mathrm{c}}$ \\
\hline Log-additive model & - & $0.98(0.61-1.59)$ & $1.00(0.62-1.62)$ & - & $1.24(0.57-2.68)$ & $1.58(0.66-3.75)$ \\
\hline MLH3 rs 175080 & $78(100)$ & & & $28(100)$ & & \\
\hline Pro/Pro & $19(24.4)$ & $1{(\text { reference })^{e}}^{e}$ & $1{(\text { reference })^{e}}^{e}$ & $3(10.7)$ & $1{(\text { reference })^{e}}^{e}$ & $1{\text { (reference })^{e}}^{e}$ \\
\hline Pro/Leu & $42(53.8)$ & $1.13(0.59-2.19)$ & $1.17(0.60-2.27)$ & $17(60.7)$ & $3.78(0.97-14.79)$ & $3.61(0.88-14.85)$ \\
\hline Leu/Leu & $17(21.8)$ & $1.17(0.53-2.61)$ & $1.20(0.54-2.68)$ & $8(28.6)$ & $4.36(0.95-20.04)$ & $4.29(0.89-20.78)$ \\
\hline Dominant model & $59(75.6)$ & $1.14(0.61-2.14)$ & $1.18(0.62-2.22)$ & $25(89.3)$ & $3.95(1.05-14.81)^{c}$ & $3.81(0.97-14.95)$ \\
\hline Recessive model & $17(21.8)$ & $1.08(0.56-2.10)$ & $1.08(0.56-2.10)$ & $8(28.6)$ & $1.64(0.57-4.69)$ & $1.67(0.55-5.02)$ \\
\hline Log-additive model & - & $1.09(0.73-1.62)$ & $1.10(0.74-1.63)$ & - & $1.93(0.97-3.86)$ & $1.93(0.93-4.01)$ \\
\hline
\end{tabular}

B, Gender

\begin{tabular}{|c|c|c|c|c|c|c|}
\hline \multirow[b]{2}{*}{ Genotype } & \multicolumn{3}{|c|}{ Male } & \multicolumn{3}{|c|}{ Female } \\
\hline & $\mathrm{n}(\%)$ & OR $(95 \% \mathrm{CI})$ & $\begin{array}{l}\text { Adjusted OR } \\
(95 \% \mathrm{CI})^{\mathrm{b}}\end{array}$ & $\mathrm{n}(\%)$ & OR $(95 \% \mathrm{CI})$ & $\begin{array}{c}\text { Adjusted } \\
\text { OR }(95 \% \mathrm{CI})^{\mathrm{b}}\end{array}$ \\
\hline MSH6 rs 1042821 & $16(100)$ & & & $90(100)$ & & \\
\hline Gly/Gly & $11(68.8)$ & 1 (reference) $^{\mathrm{e}}$ & 1 (reference) $^{\mathrm{e}}$ & $57(63.3)$ & 1 (reference) $^{\mathrm{e}}$ & 1 (reference) $^{\mathrm{e}}$ \\
\hline Gly/Glu & $4(25.0)$ & $0.86(0.21-3.54)$ & $0.96(0.20-4.52)$ & $26(28.9)$ & $0.70(0.41-1.22)$ & $0.70(0.40-1.22)$ \\
\hline Glu/Glu & $1(6.3)$ & $0.86(0.07-10.66)$ & $1.08(0.07-16.53)$ & $7(7.8)$ & $4.42(1.10-17.75)^{\mathrm{c}}$ & $4.78(1.17-19.56)^{\mathrm{c}}$ \\
\hline Dominant model & $5(31.3)$ & $0.86(0.23-3.19)$ & $0.98(0.23-4.24)$ & $33(36.7)$ & $0.86(0.51-1.44)$ & $0.86(0.51-1.45)$ \\
\hline Recessive model & $1(6.3)$ & $0.90(0.08-10.77)$ & $1.09(0.08-15.61)$ & $7(7.8)$ & $5.00(1.26-19.84)^{\mathrm{c}}$ & $5.42(1.34-21.92)^{\mathrm{c}}$ \\
\hline Log-additive model & - & $0.90(0.33-2.48)$ & $1.00(0.32-3.14)$ & - & $1.08(0.69-1.69)$ & $1.09(0.70-1.71)$ \\
\hline
\end{tabular}

C, Age

\begin{tabular}{|c|c|c|c|c|c|c|}
\hline \multirow[b]{2}{*}{ Genotype } & \multicolumn{3}{|c|}{$<50$ years } & \multicolumn{3}{|c|}{$\geq 50$ years } \\
\hline & $\mathrm{n}(\%)$ & OR $(95 \% \mathrm{CI})$ & $\begin{array}{c}\text { Adjusted } \\
\text { OR }(95 \% \mathrm{CI})^{\mathrm{b}}\end{array}$ & $\mathrm{n}(\%)$ & OR $(95 \% \mathrm{CI})$ & $\begin{array}{c}\text { Adjusted } \\
\text { OR }(95 \% \text { CI })^{\mathrm{b}}\end{array}$ \\
\hline MSH6 rs 1042821 & $42(100)$ & & & $64(100)$ & & \\
\hline Gly/Gly & $29(69.0)$ & 1 (reference) $^{\mathrm{e}}$ & 1 (reference) $^{\mathrm{e}}$ & $39(60.9)$ & 1 (reference) $^{\mathrm{e}}$ & $1{(\text { reference })^{\mathrm{e}}}$ \\
\hline Gly/Glu & $12(28.6)$ & $0.56(0.25-1.27)$ & $0.56(0.25-1.26)$ & $18(28.1)$ & $0.84(0.43-1.64)$ & $0.86(0.44-1.70)$ \\
\hline Glu/Glu & $1(2.4)$ & $0.31(0.03-2.79)$ & $0.32(0.04-2.93)$ & $7(10.9)$ & $-{ }^{\mathrm{d}}$ & $-{ }^{d}$ \\
\hline Dominant model & $13(31.0)$ & $0.53(0.24-1.16)$ & $0.53(0.24-1.17)$ & $25(39.1)$ & $1.17(0.63-2.17)$ & $1.21(0.64-2.27)$ \\
\hline Recessive model & $1(2.4)$ & $0.38(0.04-3.37)$ & $0.40(0.04-3.58)$ & $7(10.9)$ & $-{ }^{\mathrm{d}}$ & $-{ }^{\mathrm{d}}$ \\
\hline Log-additive model & - & $0.56(0.28-1.12)$ & $0.56(0.28-1.12)$ & - & $1.57(0.93-2.66)$ & $1.63(0.95-2.79)$ \\
\hline
\end{tabular}

aOnly SNPs presenting significant findings are shown. ${ }^{\mathrm{b} O R s}$ were adjusted for gender (male and female), age $(<30,30-49,50-69$, and $\geq 70$ years $)$, and smoking status (non-smoker and smoker). ${ }^{\mathrm{c} S}$ Significant results $(\mathrm{P}<0.05)$ highlighted in bold. ${ }^{\mathrm{d}}$ Genotype not found in the corresponding

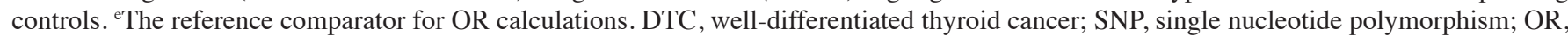
odds ratio; CI, confidence interval. 
Table V. Two-way SNP interactions: distribution of combined genotypes in the case $(\mathrm{n}=106)$ and control $(\mathrm{n}=212)$ populations and associated DTC risk (crude and adjusted ORs). ${ }^{\mathrm{a}}$

\begin{tabular}{|c|c|c|c|c|c|c|c|}
\hline \multirow[b]{3}{*}{ Genotype } & \multirow{2}{*}{\multicolumn{3}{|c|}{ Genotype frequency }} & \multicolumn{4}{|c|}{ DTC risk } \\
\hline & & & & \multirow[b]{2}{*}{ OR $(95 \% \mathrm{CI})$} & \multirow[b]{2}{*}{ P-value ${ }^{b}$} & \multirow{2}{*}{$\begin{array}{c}\text { Adjusted } \\
\text { OR }(95 \% \mathrm{CI})^{\mathrm{c}}\end{array}$} & \multirow[b]{2}{*}{ P-value } \\
\hline & Controls n (\%) & Cases n $(\%)$ & P-value ${ }^{b}$ & & & & \\
\hline $\begin{array}{l}\text { MSH6 rs } 1042821-M S H 3 \\
\text { rs26279 }\end{array}$ & $209(100)$ & $105(100)$ & 0.167 & & & & \\
\hline Gly/Gly-Thr/Thr & $59(28.2)$ & $29(27.6)$ & & 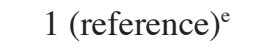 & & 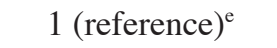 & \\
\hline Gly/Gly-Thr/Ala & $56(26.8)$ & $32(30.5)$ & & $1.16(0.62-2.16)$ & 0.64 & $1.18(0.63-2.20)$ & 0.62 \\
\hline Gly/Glu-Thr/Thr & $31(14.8)$ & $16(15.2)$ & & $1.05(0.50-2.22)$ & 0.90 & $1.14(0.53-2.43)$ & 0.74 \\
\hline Gly/Glu-Thr/Ala & $33(15.8)$ & $10(9.5)$ & & $0.62(0.27-1.42)$ & 0.26 & $0.60(0.26-1.39)$ & 0.23 \\
\hline Gly/Gly-Ala/Ala & $11(5.3)$ & $7(6.7)$ & & $1.30(0.46-3.69)$ & 0.63 & $1.26(0.44-3.62)$ & 0.67 \\
\hline Glu/Glu-Thr/Thr & $5(2.4)$ & $8(7.6)$ & & $3.26(0.98-10.84)$ & 0.05 & $3.81(1.11-13.13)^{d}$ & $0.03^{d}$ \\
\hline \multicolumn{8}{|l|}{ Glu/Glu-Thr/Ala } \\
\hline \multicolumn{8}{|l|}{ Glu/Glu-Ala/Ala } \\
\hline Gly/Glu-Ala/Ala & $14(6.7)$ & $3(2.9)$ & & $0.44(0.12-1.64)$ & 0.22 & $0.42(0.11-1.59)$ & 0.20 \\
\hline \multicolumn{7}{|l|}{ rs1042821 } & \\
\hline Pro/Pro-Gly/Gly & $32(15.2)$ & $19(17.9)$ & & 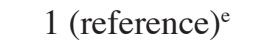 & & 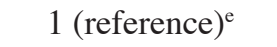 & \\
\hline Pro/Pro-Gly/Glu & $26(12.4)$ & $2(1.9)$ & & $0.13(0.03-0.61)^{d}$ & $0.01^{\mathrm{d}}$ & $0.11(0.02-0.53)^{d}$ & $0.01^{\mathrm{d}}$ \\
\hline Pro/Leu-Gly/Gly & $71(33.8)$ & $36(34.0)$ & & $0.85(0.43-1.71)$ & 0.66 & $0.81(0.40-1.65)$ & 0.56 \\
\hline Pro/Leu-Gly/Glu & $35(16.7)$ & $19(17.9)$ & & $0.91(0.41-2.03)$ & 0.83 & $0.94(0.41-2.13)$ & 0.88 \\
\hline Pro/Pro-Glu/Glu & $5(2.4)$ & $8(7.5)$ & & $2.70(0.77-9.44)$ & 0.12 & $3.09(0.85-11.27)$ & 0.09 \\
\hline \multicolumn{8}{|l|}{ Pro/Leu-Glu/Glu } \\
\hline \multicolumn{8}{|l|}{ Leu/Leu-Glu/Glu } \\
\hline Leu/Leu-Gly/Gly & $24(11.4)$ & $13(12.3)$ & & $0.91(0.38-2.20)$ & 0.84 & $0.83(0.34-2.03)$ & 0.68 \\
\hline Leu/Leu-Gly/Glu & $17(8.1)$ & $9(8.5)$ & & $0.89(0.33-2.39)$ & 0.82 & $0.89(0.33-2.43)$ & 0.82 \\
\hline $\begin{array}{l}\text { MSH4 rs5745325-MSH6 } \\
\text { rs1042821 }\end{array}$ & $210(100)$ & \multicolumn{6}{|c|}{ rs 1042821} \\
\hline Ala/Ala-Gly/Gly & $53(25.2)$ & $36(34.0)$ & & 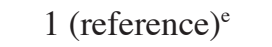 & & 1 (reference) $^{\mathrm{e}}$ & \\
\hline Ala/Ala-Gly/Glu & $41(19.5)$ & $20(18.9)$ & & $0.72(0.36-1.42)$ & 0.34 & $0.74(0.37-1.47)$ & 0.39 \\
\hline Ala/Thr-Gly/Gly & $59(28.1)$ & $26(24.5)$ & & $0.65(0.35-1.21)$ & 0.18 & $0.66(0.35-1.23)$ & 0.19 \\
\hline Ala/Thr-Gly/Glu & $30(14.3)$ & 7 (6.6) & & $0.34(0.14-0.87)^{d}$ & $0.02^{d}$ & $0.35(0.14-0.88)^{d}$ & $\mathbf{0 . 0 3}{ }^{\mathrm{d}}$ \\
\hline Ala/Ala-Glu/Glu & $12(5.7)$ & $11(10.4)$ & & $1.35(0.54-3.39)$ & 0.52 & $1.43(0.56-3.66)$ & 0.45 \\
\hline \multicolumn{8}{|l|}{ Ala/Thr-Glu/Glu } \\
\hline \multicolumn{8}{|l|}{ Thr/Thr-Gly/Glu } \\
\hline \multicolumn{8}{|l|}{ Thr/Thr-Glu/Glu } \\
\hline Thr/Thr-Gly/Gly & $15(7.1)$ & $6(5.7)$ & & $0.59(0.21-1.66)$ & 0.32 & $0.60(0.21-1.70)$ & 0.33 \\
\hline
\end{tabular}

data not shown). Also, as depicted in Table IV, the homozygous variant genotype of this SNP was found to confer increased DTC risk in females only, under both the co-dominant $(\mathrm{OR}=4.42,95 \% \mathrm{CI}: 1.10-17.75, \mathrm{P}=0.04$ and adjusted $\mathrm{OR}=4.78$, 95\% CI: $1.17-19.56, \mathrm{P}=0.03)$ and the recessive model $(\mathrm{OR}=5.00$, 95\% CI: $1.26-19.84, \mathrm{P}=0.02$ and adjusted $\mathrm{OR}=5.42,95 \% \mathrm{CI}$ : $1.34-21.92, \mathrm{P}=0.02)$, supporting the idea that this polymorphism might influence DTC susceptibility, particularly in women. The study population was also stratified according to the age of diagnosis (Table IV). In order to avoid excessively low numbers in each strata, only two groups were formed: $<50$ and $\geq 50$ years. In the elderly group ( $\geq 50$ years), a highly significant difference in the frequency distribution of MSH6 rs1042821 genotypes was observed between DTC patients and the corresponding controls $(\mathrm{P}=0.001$ in the codominant model, data not shown). Unfortunately, no MSH6 rs1042821 
homozygous variant individuals $\geq 50$ years were observed in the control group, limiting OR calculations and subsequent analysis for this SNP. Further analysis of our study subjects after histological type, gender and age stratification revealed no other significant correlations between the analysed SNPs and DTC risk.

Proteins that participate in a common DNA repair pathway functionally interact with each other, establishing ground for additive or even multiplicative effects of different SNPs of the same pathway on DNA repair activity and, hence, cancer risk. This has been previously demonstrated for other DNA repair pathways $(16,44,45)$ and, most likely, also applies to MMR, justifying the usefulness of assessing the effect of combined genotypes on DTC risk. As detailed in Table V, the combined genotype distribution of the MSH6 rs1042821-MLH3 rs175080 SNP pair was significantly different in cases and controls $(\mathrm{P}=0.032)$. On logistic regression analysis, when MSH6 rs1042821 and MSH3 rs26279 were considered together, a significantly increased risk was observed in the pooled group of MSH6 rs1042821 variant allele homozygotes, despite only after adjusting for gender, age and smoking status (adjusted $\mathrm{OR}=3.81,95 \% \mathrm{CI}: 1.11-13.13, \mathrm{P}=0.03)$. Interestingly, two other MSH6 rs1042821 genotype combinations, all involving the rs1042821 heterozygous genotype, yielded significant results in the opposite direction: a significantly decreased risk was detected in combined MSH6 rs1042821-MSH4 rs5745325 heterozygotes $(\mathrm{OR}=0.34,95 \% \mathrm{CI}: 0.14-0.87, \mathrm{P}=0.02$ and adjusted $\mathrm{OR}=0.35,95 \% \mathrm{CI}: 0.14-0.88, \mathrm{P}=0.03$ ), as well as in individuals simultaneously heterozygous for MSH6 rs1042821 and homozygous for the common allele of $M L H 3$ rs 175080 $(\mathrm{OR}=0.13,95 \% \mathrm{CI}: 0.03-0.61, \mathrm{P}=0.01$ and adjusted $\mathrm{OR}=0.11$, $95 \% \mathrm{CI}: 0.02-0.53, \mathrm{P}=0.01)$. None of the remaining genotype combinations showed association with disease (data not shown).

Finally, since MSH3 and MLH3 (whose studied variants yielded significant associations on SNP pair analysis) are located in the same chromosomic region that DTC markers reported in prior GWAS (rs13184587 at 5q14.1 and rs10136427 at $14 q 24.3$, respectively) (13), we used LDLink (43) to verify in silico any potential linkage disequilibrium relation between these MMR variants and the GWAS-suggested markers co-localized in the same chromosomic region. No linkage disequilibrium was observed between either MSH3 rs26279 and rs13184587 or MLH3 rs175080 and rs10136427 in European populations (data not shown).

\section{Discussion}

To our knowledge, this was the first study evaluating the potential role of MMR SNPs on DTC susceptibility in Caucasian populations.

We observed a significantly increased DTC risk in MSH6 rs1042821 variant allele homozygotes (Glu/Glu). MSH6 rs1042821 is probably one of the most studied SNPs of the MMR pathway and its potential association with cancer (other than TC) has previously been addressed, with inconsistent results: rs1042821 has been associated with increased CRC risk $(46,47)$, as well as with triple negative breast cancer (TNBC) (48) and highly malignant bladder cancer (49). Contrasting results have been reported for hepatocellular (50), colorectal (51) and pancreatic cancer (52). Most studies, however, present inconclusive findings (53-57), including a recent meta-analysis (58) aggregating data from many of the above-quoted studies. It is possible that organ and population-specific characteristics (e.g., genetic background and environmental exposure) may have contributed to such diverse observations. More recently, rs1042821 has also been detected through sequencing techniques in several cancer cases (59-61) but, considering the high population frequency of this SNP, this could be merely coincidental.

The involvement of MSH6 SNPs in cancer susceptibility (DTC, in particular) is expected for three fundamental reasons. Firstly, because it is biologically plausible: MSH6 integrates the MutS $\alpha$ complex, a sensor of genetic damage that, besides its role in the repair of replication errors, cooperates with other DNA repair and damage-response signalling pathways to allow for cell cycle arrest, DNA repair and/or apoptosis of genetically damaged cells. Of importance for DTC susceptibility, MutS $\alpha$ ensures accurate homologous recombination repair of double strand breaks and cooperates with MUTYH in the repair of 8-oxoGuanine [reviewed in (27-29)], lesions that commonly arise from IR exposure, the most well-known DTC risk factor. Secondly, because of the functional impact of MSH6 mutations: Experimental studies in MSH6-deficient yeast, mice or human cells demonstrate that MSH6 mutations results in partial MMR deficiency (mild mutator phenotype, characterized by weak microsatellite instability, MSI-L) and increased cancer susceptibility in animal models (27). And finally because, in the clinical context, MSH6 mutations are associated with cancer syndromes (and TC, possibly): Inherited MSH6 germline mutations are responsible for $7-10 \%$ of LS cases, patients presenting an atypical phenotype (lower CRC incidence-with later onset-high incidence of endometrial cancer and lower degree or absence of MSI), compared to the more frequent $M S H 2$ and $M L H 1$-mutant LS cases $(27,31,39,62)$. TC-despite not part of the usual LS spectrum- has been sporadically observed in LS patients harbouring $M S H 2$ and $M L H 1$ mutations (36,38-40) and, more recently, also in a MSH6-mutant LS case (37). MSH6 mutations were also recently observed in both anaplastic (33) and papillary TC (34). In the latter study, MSH6 was even the most frequently mutated gene and two of these mutations (Gly355Ser and Ala36Val) were coincidental in family-related patients, suggesting a causative association. For all of the above, it is likely that MSH6 genetic variation contributes to TC development.

The rs1042821 SNP is a common missense variant that involves the substitution $116 \mathrm{G}>\mathrm{A}$ in exon 1 of the MSH6 gene. It results in the substitution of glutamic acid for glycine at position 39 (Gly39Glu) of the MSH6 N-terminal region (NTR), a highly disordered domain upstream of the mismatch binding domain. The importance of the MSH6 NTR is being increasingly recognized as missense mutations in this region have been associated with cancer [an exhaustive list of LS-associated mutations is available in the InSiGHT database (32)]. Interestingly, the MSH6 NTR is absent from prokaryotic MutS which, coincidentally, does not share some of the functions of eukaryotic MutS $\alpha$ (e.g., activation of apoptosis) (63), suggesting a critical role for this region in such processes. As extensively reviewed 
in Edelbrock et al (28), several sequence motifs in the NTR may be of relevance to the multitude of actions performed by MSH6, including: 1) a short, conserved PCNA interacting protein (PIP) motif, located near the N-terminal extreme, that allows PCNA binding; 2) a PWWP sequence motif, distal to the PIP box, that mediates interactions with chromatin and chromatin-associating proteins; 3 ) a conserved motif near the NTR C-terminus, rich in positively charged amino acids that (through electrostatic attraction) contributes to nonspecific DNA binding and stabilizes the MutS $\alpha$-DNA interaction (possibly modulating the residence time of MutS $\alpha$ at the lesion site); 4) nuclear localization sequences (NLSs, e.g., a conserved Ser-Pro-Ser sequence-amino acids 41-43-containing phosphorylated serines), that may contribute to the nuclear import of MutS $\alpha$; and 5) multiple phosphorylation sites (19 out of the 23 identified in MSH6, according to the updated list at http://www.uniprot.org/uniprot/P52701), that may be involved in the post-translational regulation of MutS $\alpha$ stability, nuclear import and differential downstream signalling for MMR and DNA damage response. The NTR may also be responsible for other protein interactions.

Functional assays are needed to confirm if the MSH6 rs1042821 variant affects the function of MutS $\alpha$. However, given its location-in the MSH6 NTR, near a NLS containing two phosphorylation sites (Ser41 and Ser43)- it is possible that rs1042821 interferes with phosphorylation of these residues (both MAPK recognition motifs) and hence with the post-translational regulation of MutS $\alpha$ stability, nuclear import or activity (28). The rs1042821 SNP may also interfere with non-specific DNA binding since glutamate, contrasting with glycine, is negatively charged at physiological $\mathrm{pH}$. This may hamper electrostatic attraction to the phosphate backbone of DNA, interfere with the stability of the MutS $\alpha$-DNA interaction and hence decrease the residence time of MutS $\alpha$ at the lesion site [previously suggested to play a role in the differential regulation of the DNA repair and apoptosis signalling roles of MSH6 (63)]. In fact, increasing number of negatively charged glutamate residues within the amino acid 231-289 NTR segment of the yeast Msh6 increases mutation rates in these cells (64) and substitutions of glutamic acid for glycine, in general, can determine the formation of sterically different helical structures, polypeptide folding, and intrinsic aggregation (51). Whether this applies to MSH6 rs1042821 remains to be established.

In our study, upon stratification, the association between the MSH6 rs1042821 homozygous variant genotype and increased DTC risk was especially evident for the follicular histotype, female sex and, possibly, older age ( $\geq 50$ years). Concerning the histological type of tumour, this contrasts with prior evidence: rs1042821 has been associated with the development of $B R A F$ mutated (Val600Glu) colon tumours (54) -only in microsatellite stable (MSS), not MSI tumours- and the Val600Glu BRAF mutation is a hallmark of papillary, not follicular TC (3). However, as in our study, this observation resulted from stratification analysis with only a limited number of subjects in each strata $[\mathrm{n}=3$ for follicular TC cases with Glu/Glu genotype in our study; $\mathrm{n}=4$ for $B R A F$ mutated, MSS tumours with Glu/Glu genotype in (54)]. Either observation could therefore be due to chance (type I statistical error), hampering solid conclusions. Further studies with a larger sample size are needed to clarify the relationship, if any, between rs1042821 and DTC histological type. The genotype-disease association was stronger among women, an expected finding since DTC affects women more than men $(1,2)$, differential incidence starting with the onset of puberty and declining after menopause (65). Also, TC rates in women with breast cancer history (and vice-versa) are higher than expected (66), suggesting some common ground between these conditions. Oestrogen could be the "missing link': Besides its well-established role in the pathogenesis of several endocrine-related cancers (e.g., breast, endometrial, ovarian) (67), oestrogen may promote the growth of TC cells and thus contribute to development and progression of DTC, through increased transcription of ERE-containing genes, activation of the MAPK and PI3K signalling pathways, modulation of the TC microenvironment or specific effects on thyroid stem and progenitor cells (65). Oestrogen has also been suggested to give rise to cancer-initiating mutations through the formation of DNA adducts and other oxidative lesions, high levels of which have been observed in women with breast, thyroid or ovarian cancer (68). On the other hand, MSH6 is increasingly being implicated in such oestrogen-associated cancers as 1) in vitro oestrogen exposure after catechol-O-methyltransferase inhibition increases the levels of 8-oxo-dG (69), an oxidative DNA lesion whose repair involves the MutS $\alpha$ complex; 2) MSH6 mutations and reduced MSH6 mRNA expression have been reported in breast cancer patients and breast tumour derived cell lines, respectively (70); 3) in LS patients, endometrial cancer is commonly associated with MSH6 mutations $(27,31,39,62)$; 4) MSH2- the binding partner of MSH6 in MutS $\alpha$ - is able to transactivate the oestrogen receptor $\alpha$, through its MSH6 interaction domain (71); 5) several DNA repair SNPs have been associated with increased oestrogen sensitivity in the development of breast cancer (72-74). Also, we previously reported a non-significant breast cancer risk increase in rs1042821 variant allele homozygotes (53), in line with the results reported here. Overall, if oestrogen indeed contributes to DTC and MSH6 is indeed involved in oestrogen-associated cancers, it is only logical that a putative association between rs1042821 and DTC susceptibility is particularly visible in women. Finally, in the current study, considering only individuals of age $\geq 50$ years, the rs1042821 homozygous variant genotype was detected only in DTC patients, not in controls. This may suggest that rs1042821 is associated with DTC susceptibility particularly among older individuals. This is compatible with the observation of later onset cancer in LS patients harbouring MSH6 mutations $(27,31,39)$. Also, in line with our results, rs1042821 has been associated with increased breast cancer risk in women of age $>60$ years and decreased risk in women of age $\leq 60$ years (57). Finally, MSH6 has been demonstrated to be markedly downregulated in senescent cells (75), suggesting that MutS $\alpha$ activity decreases during the aging process. Since our results were based on stratified analysis, further studies are required to confirm this finding.

On paired SNP analysis, several associations were observed in our study. Most of these involve MSH6 rs1042821, possibly reflecting an individual SNP effect. However- since MMR proteins functionally interact within the same pathwayan additive (or even multiplicative) effect with other MMR SNPs is possible. Supporting this hypothesis, several studies 
have shown that, although individual susceptibility alleles may have only a modest effect, DTC risk may be substantially increased when multiple risk variants are considered together $(15,16)$. Considering the strong genetic component of DTC susceptibility, such a role for gene-gene interactions is likely (16). One SNP combination comprising the MSH6 rs1042821 homozygous variant genotype was associated with increased DTC risk, as expected from single SNP analysis. Interestingly, two other SNP pairs containing the MSH6 heterozygous genotype were associated with a risk reduction. A similar non-significant trend was already evident on single SNP analysis, suggesting a protective effect for the MSH6 rs1042821 heterozygous genotype. Prior evidence supports this hypothesis: We previously reported a breast cancer risk reduction in combined $M S H 6$ rs1042821 heterozygotes/MSH3 rs26279 common allele homozygotes (53).

Other studies $(50-53,57)$, including a recent meta-analysis by Li et al (58), detected a cancer risk reduction in MSH6 rs1042821 heterozygotes or variant allele carriers. It should be noted that these observations in variant allele carriers do not contradict our prior suggestion of risk increase in variant homozygotes: considering, as stated above, i) the dual role of MSH6 on DNA repair and apoptosis; ii) the likely involvement of the MSH6 NTR in the differential regulation of such functions; and iii) the location and potential impact of rs1042821, it is possible that this SNP has distinct effects on each of MSH6 functions (DNA repair or apoptosis signalling) critically impairing one but somehow favouring the other. If so, variant allele homozygotes-lacking the common form of MSH6-could have higher cancer risk, while the presence of both forms in heterozygotes could be of some benefit. Further studies are required to confirm this hypothesis. Furthermore, it is interesting to note that two out of the three SNP pairs significantly associated with DTC susceptibility in our study involve variants that are located in the exact same chromosomic region of previously GWAS-suggested DTC markers. According to our in silico analysis, no linkage disequilibrium was identified between these co-localized variants in European populations. However, since it was not possible to verify this hypothesis with experimental data from our study, we cannot exclude the possibility that some of the variants analysed are indeed in linkage disequilibrium with previously suggested DTC markers, in the Portuguese population.

In conclusion, the rapidly increasing incidence of DTC (1) has prompted research on the genetic predisposing factors of this disease. Recently performed GWAS (6-12) have provided valuable contribution but, even so, explain only part of the estimated heritability of DTC $(11,15,16)$. Several reasons may contribute: it is possible that the highly stringent criteria applied to GWAS to prevent false-positive findings result in the exclusion of SNPs truly associated with DTC risk (14). Furthermore, evidence from the latest GWAS (10-13) suggests the existence of population-specific DTC risk alleles, raising the possibility that novel cancer susceptibility markers, specific for geographically distinct populations, may remain to be identified. Finally, gene-gene and gene-environment interactions, despite seldom addressed, may also play an important role and explain part of the unresolved heritability of DTC susceptibility $(15,16)$. The identification of additional common variants, gene-environment and gene-gene interactions predisposing to DTC may thus unveil at least part of the unexplained genetic component of DTC susceptibility. Hypothesis-driven case control association studies remain a valid approach and, as recently demonstrated (14), could provide valuable insight into the genetic risk factors for DTC.

This work suggests an involvement of MMR SNPs such as MSH6 rs1042821, alone or in combination, on DTC susceptibility. However, despite the care put to avoid selection bias and variant misclassification, our results should be regarded solely as a proof of concept on the role of MMR genes on DTC susceptibility. Also, since the information that was collected from study participants on prior IR exposure was not suitable for rigorous statistical analysis, it was not possible to include it as a covariate in the adjustment statistical model. Since IR exposure remains the best-established risk factor for TC, future studies should be designed in order to account for this. Finally, since no SNP functional assessment was performed, we cannot exclude the possibility that the associations observed are due to other variants, in LD with the ones considered here. Therefore, in order to obtain conclusive evidence, these preliminary findings must be replicated in larger, multicentric studies with independent datasets of patients. Such studies should be powered to allow for more sophisticated analysis (e.g., haplotype analysis, evaluation of gene-gene and gene-environment interactions), for the study of other (e.g., less frequent but potentially relevant) variants and their potential association with mutational events that occur early in DTC carcinogenesis.

\section{Acknowledgements}

The authors acknowledge the invaluable contributions of their late colleague Professor Jorge Gaspar (1963-2015). The authors wish to thank Ana Paula Azevedo and Isabel Manita for technical support. This study was supported by funding through project UID/BIM/00009/2016 [Centre for Toxicogenomics and Human Health (ToxOmics)], from Fundação para a Ciência e a Tecnologia (FCT), Portugal.

\section{References}

1. Kitahara CM and Sosa JA: The changing incidence of thyroid cancer. Nat Rev Endocrinol 12: 646-653, 2016.

2. Ferlay J, Soerjomataram I, Ervik M, Dikshit R, Eser S, Mathers C, Rebelo M, Parkin DM, Forman D and Bray F: GLOBOCAN 2012 v1.0, Cancer Incidence and Mortality Worldwide: IARC CancerBase No. 11 (Internet). International Agency for Research on Cancer, Lyon, 2013. http://globocan.iarc. fr/Default.aspx.

3. DeLellis RA and Williams ED: Tumours of the thyroid and parathyroid. In: WHO Classification of Tumours: Pathology and Genetics of Tumours of Endocrine Organs. DeLellis RA, Lloyd RV, Heitz PU and Eng C (eds). IARC Press, Lyon, pp51-56, 2004.

4. Marcello MA, Malandrino P, Almeida JF, Martins MB, Cunha LL, Bufalo NE, Pellegriti G and Ward LS: The influence of the environment on the development of thyroid tumors: A new appraisal. Endocr Relat Cancer 21: T235-T254, 2014.

5. Landa I and Robledo M: Association studies in thyroid cancer susceptibility: Are we on the right track? J Mol Endocrinol 47: R43-R58, 2011.

6. Gudmundsson J, Sulem P, Gudbjartsson DF, Jonasson JG, Masson G, He H, Jonasdottir A, Sigurdsson A, Stacey SN, Johannsdottir H, et al: Discovery of common variants associated with low TSH levels and thyroid cancer risk. Nat Genet 44: 319-322, 2012. 
7. Gudmundsson J, Sulem P, Gudbjartsson DF, Jonasson JG, Sigurdsson A, Bergthorsson JT, He H, Blondal T, Geller F, Jakobsdottir M, et al: Common variants on 9q22.33 and 14q13.3 predispose to thyroid cancer in European populations. Nat Genet 41: 460-464, 2009.

8. Gudmundsson J, Thorleifsson G, Sigurdsson JK, Stefansdottir L, Jonasson JG, Gudjonsson SA, Gudbjartsson DF, Masson G, Johannsdottir H, Halldorsson GH, et al: A genome-wide association study yields five novel thyroid cancer risk loci. Nat Commun 8: 14517, 2017.

9. Takahashi M, Saenko VA, Rogounovitch TI, Kawaguchi T, Drozd VM, Takigawa-Imamura H, Akulevich NM, Ratanajaraya C, MitsutakeN, Takamura N, etal: The FOXE1 locus is a major genetic determinant for radiation-related thyroid carcinoma in Chernobyl. Hum Mol Genet 19: 2516-2523, 2010.

10. Köhler A, Chen B, Gemignani F, Elisei R, Romei C, Figlioli G, Cipollini M, Cristaudo A, Bambi F, Hoffmann P, et al: Genome-Wide Association Study on Differentiated Thyroid Cancer. J Clin Endocrinol Metab 98: E1674-E1681, 2013.

11. Mancikova V, Cruz R, Inglada-Pérez L, Fernández-Rozadilla C, Landa I, Cameselle-Teijeiro J, Celeiro C, Pastor S, Velázquez A, Marcos R, et al: Thyroid cancer GWAS identifies 10q26.12 and $6 \mathrm{q} 14.1$ as novel susceptibility loci and reveals genetic heterogeneity among populations. Int J Cancer 137: 1870-1878, 2015.

12. Son HY, Hwangbo Y, Yoo SK, Im SW, Yang SD, Kwak SJ, Park MS, Kwak SH, Cho SW, Ryu JS, et al: Genome-wide association and expression quantitative trait loci studies identify multiple susceptibility loci for thyroid cancer. Nat Commun 8: 15966, 2017.

13. Figlioli G, Köhler A, Chen B, Elisei R, Romei C, Cipollini M, Cristaudo A, Bambi F, Paolicchi E, Hoffmann P, et al: Novel genome-wide association study-based candidate loci for differentiated thyroid cancer risk. J Clin Endocrinol Metab 99: E2084-E2092, 2014.

14. Figlioli G, Elisei R, Romei C, Melaiu O, Cipollini M, Bambi F, Chen B, Köhler A, Cristaudo A, Hemminki K, et al: A comprehensive meta-analysis of case-control association studies to evaluate polymorphisms associated with the risk of differentiated thyroid carcinoma. Cancer Epidemiol Biomarkers Prev 25: 700-713, 2016.

15. Figlioli G, Chen B, Elisei R, Romei C, Campo C, Cipollini M Cristaudo A, Bambi F, Paolicchi E, Hoffmann P, et al: Novel genetic variants in differentiated thyroid cancer and assessment of the cumulative risk. 5: 8922, 2015.

16. Landa I, Boullosa C, Inglada-Pérez L, Sastre-Perona A, Pastor S, Velázquez A, Mancikova V, Ruiz-Llorente S, Schiavi F, Marcos R, et al: An Epistatic Interaction between the PAX8 and STK17B genes in papillary thyroid cancer susceptibility. PLoS One 8: e74765, 2013.

17. Adjadj E, Schlumberger M and de Vathaire F: Germ-line DNA polymorphisms and susceptibility to differentiated thyroid cancer. Lancet Oncol 10: 181-190, 2009.

18. Ho T, Li G, Lu J, Zhao C, Wei Q and Sturgis EM: Association of XRCC1 polymorphisms and risk of differentiated thyroid carcinoma: A case-control analysis. Thyroid 19: $129-135,2009$

19. Santos LS, Branco SC, Silva SN, Azevedo AP, Gil OM, Manita I, Ferreira TC, Limbert E, Rueff J and Gaspar JF: Polymorphisms in base excision repair genes and thyroid cancer risk. Oncol Rep 28: 1859-1868, 2012.

20. Silva SN, Gil OM, Oliveira VC, Cabral MN, Azevedo AP, Faber A, Manita I, Ferreira TC, Limbert E, Pina JE, et al: Association of polymorphisms in ERCC2 gene with non-familial thyroid cancer risk. Cancer Epidemiol Biomarkers Prev 14: 2407-2412, 2005.

21. Santos LS, Gomes BC, Gouveia R, Silva SN, Azevedo AP, Camacho V, Manita I, Gil OM, Ferreira TC, Limbert E, et al: The role of CCNH Val270Ala (rs2230641) and other nucleotide excision repair polymorphisms in individual susceptibility to well-differentiated thyroid cancer. Oncol Rep 30: 2458-2466, 2013.

22. Gomes BC, Silva SN, Azevedo AP, Manita I, Gil OM, Ferreira TC, Limbert E, Rueff J and Gaspar JF: The role of common variants of non-homologous end-joining repair genes XRCC4, LIG4 and Ku80 in thyroid cancer risk. Oncol Rep 24: 1079-1085, 2010.

23. Rahimi M, Fayaz S, Fard-Esfahani A, Modarressi MH, Akrami SM and Fard-Esfahani P: The role of Ile3434Thr XRCC7 gene polymorphism in differentiated thyroid cancer risk in an Iranian population. Iran Biomed J 16: 218-222, 2012.
24. Xu L, Doan PC, Wei Q, Liu Y, Li G and Sturgis EM: Association of BRCA1 functional single nucleotide polymorphisms with risk of differentiated thyroid carcinoma. Thyroid 22: 35-43, 2012.

25. Siraj AK, Al-Rasheed M, Ibrahim M, Siddiqui K, Al-Dayel F, Al-Sanea O, Uddin S and Al-Kuraya K: RAD52 polymorphisms contribute to the development of papillary thyroid cancer susceptibility in middle eastern population. J Endocrinol Invest 31: 893-899, 2008.

26. Bastos HN, Antão MR, Silva SN, Azevedo AP, Manita I, Teixeira V, Pina JE, Gil OM, Ferreira TC, Limbert E, et al: Association of polymorphisms in genes of the homologous recombination DNA repair pathway and thyroid cancer risk. Thyroid 19: 1067-1075, 2009.

27. Hsieh $P$ and Yamane K: DNA mismatch repair: Molecular mechanism, cancer and ageing. Mech Ageing Dev 129: 391-407, 2008.

28. Edelbrock MA, Kaliyaperumal S and Williams KJ: Structural, molecular and cellular functions of MSH2 and MSH6 during DNA mismatch repair, damage signaling and other noncanonical activities. Mutat Res 743-744: 53-66, 2013.

29. Bridge G, Rashid S and Martin S: DNA Mismatch repair and oxidative DNA damage: Implications for cancer biology and treatment. Cancers (Basel) 6: 1597-1614, 2014.

30. Li Z, Pearlman AH and Hsieh P: DNA mismatch repair and the DNA damage response. DNA Repair(Amst) 38: 94-101, 2016

31. Peltomäki P: Update on Lynch syndrome genomics. Fam Cancer 15: 385-393, 2016

32. Thompson BA, Spurdle AB, Plazzer JP, Greenblatt MS, Akagi K, Al-Mulla F, Bapat B, Bernstein I, Capellá G, den Dunnen JT, et al: Application of a 5-tiered scheme for standardized classification of 2,360 unique mismatch repair gene variants in the InSiGHT locus-specific database. Nat Genet 46: 107-115, 2014

33. Kunstman JW, Juhlin CC, Goh G, Brown TC, Stenman A, Healy JM, Rubinstein JC, Choi M, Kiss N, Nelson-Williams C, et al: Characterization of the mutational landscape of anaplastic thyroid cancer via whole-exome sequencing. Hum Mol Genet 24: 2318-2329, 2015.

34. Yu Y, Dong L, Li D, Chuai S, Wu Z, Zheng X, Cheng Y, Han L, Yu J and Gao M: Targeted DNA sequencing detects mutations related to susceptibility among familial non-medullary thyroid cancer. Sci Rep 5: 16129, 2015.

35. Xu B and Ghossein R: Genomic landscape of poorly differentiated and anaplastic thyroid carcinoma. Endocr Pathol 27: 205-212, 2016.

36. Broaddus RR, Lynch PM, Lu KH, Luthra R and Michelson SJ: Unusual tumors associated with the hereditary nonpolyposis colorectal cancer syndrome. Mod Pathol 17: 981-989, 2004

37. Johnson JM, Chen J, Ali SM, Dardi IK, Tuluc M, Cognetti D, Campling B and Sama AR: Molecular profiling of synchronous colon cancers and anaplastic thyroid cancer in a patient with lynch syndrome. J Gastrointest Cancer: Oct 6, 2016 (Epub ahead of print).

38. Pande M, Wei C, Chen J, Amos CI, Lynch PM, Lu KH Lucio LA, Boyd-Rogers SG, Bannon SA, Mork ME and Frazier ML: Cancer spectrum in DNA mismatch repair gene mutation carriers: Results from a hospital based Lynch syndrome registry. Fam Cancer 11: 441-447, 2012.

39. Stulp RP, Herkert JC, Karrenbeld A, Mol B, Vos YJ and Sijmons RH: Thyroid cancer in a patient with a germline MSH2 mutation. Case report and review of the Lynch syndrome expanding tumour spectrum. Hered Cancer Clin Pract 6: 15-21, 2008.

40. Pelizzo MR, Pennelli G, Zane M, Galuppini F, Colletti PM, Merante Boschin I and Rubello D: Papillary thyroid carcinoma (PTC) in Lynch syndrome: Report of two cases and discussion on Lynch syndrome behaviour and genetics. Biomed Pharmacother 74: 9-16, 2015.

41. Gatzidou E, Michailidi C, Tseleni-Balafouta S and Theocharis S: An epitome of DNA repair related genes and mechanisms in thyroid carcinoma. Cancer Lett 290: 139-147, 2010 .

42. Solé X, Guinó E, Valls J, Iniesta $\mathrm{R}$ and Moreno V: SNPStats: A web tool for the analysis of association studies. Bioinformatics 22: 1928-1929, 2006

43. Machiela MJ and Chanock SJ: LDlink: A web-based application for exploring population-specific haplotype structure and linking correlated alleles of possible functional variants. Bioinformatics 31: 3555-3557, 2015. 
44. Han W, Kim KY, Yang SJ, Noh DY, Kang D and Kwack K: SNP-SNP interactions between DNA repair genes were associated with breast cancer risk in a Korean population. Cancer 118: 594-602, 2012

45. Sapkota Y, Mackey JR, Lai R, Franco-Villalobos C Lupichuk S, Robson PJ, Kopciuk K, Cass CE, Yasui Y and Damaraju S: Assessing SNP-SNP interactions among DNA Repair, modification and metabolism related pathway genes in breast cancer susceptibility. PLoS One 8: e64896, 2013.

46. Berndt SI, Platz EA, Fallin MD, Thuita LW, Hoffman SC and Helzlsouer KJ: Mismatch repair polymorphisms and the risk of colorectal cancer. Int J Cancer 120: 1548-1554, 2007.

47. Campbell PT, Curtin K, Ulrich CM, Samowitz WS, Bigler J, Velicer CM, Caan B, Potter JD and Slattery ML: Mismatch repair polymorphisms and risk of colon cancer, tumour microsatellite instability and interactions with lifestyle factors. Gut 58: 661-667, 2009

48. Lee E, Levine EA, Franco VI, Allen GO, Gong F, Zhang $\mathrm{Y}$ and $\mathrm{Hu} \mathrm{JJ}$ : Combined genetic and nutritional risk models of triple negative breast cancer. Nutr Cancer 66: 955-963, 2014.

49. Sanyal S, De Verdier PJ, Steineck G, Larsson P, Onelöv E, Hemminki K and Kumar R: Polymorphisms in XPD, XPC and the risk of death in patients with urinary bladder neoplasms. Acta Oncol 46: 31-41, 2007.

50. Liu Y, Zhang X, Jia J, Tang L, Gao X, Yan L, Wang L, Yu F, Ma N, Liu W, et al: Correlation between polymorphisms in DNA mismatch repair genes and the risk of primary hepatocellular carcinoma for the Han population in northern China. Scand J Gastroenterol 50: 1404-1410, 2015.

51. Tulupova E, Kumar R, Hanova M, Slyskova J, Pardini B, Polakova V, Naccarati A, Vodickova L, Novotny J, Halamkova J, et al: Do polymorphisms and haplotypes of mismatch repair genes modulate risk of sporadic colorectal cancer? Mutat Res 648: 40-45, 2008.

52. Dong X, Li Y, Chang P, Hess KR, Abbruzzese JL and Li D DNA mismatch repair network gene polymorphism as a susceptibility factor for pancreatic cancer. Mol Carcinog 51: 491-499, 2012

53. Conde J, Silva SN, Azevedo AP, Teixeira V, Pina JE, Rueff J and Gaspar JF: Association of common variants in mismatch repair genes and breast cancer susceptibility: A multigene study. BMC Cancer 9: 344, 2009.

54. Curtin K, Samowitz WS, Wolff RK, Caan BJ, Ulrich CM, Potter JD and Slattery ML: MSH6 G39E polymorphism and CpG island methylator phenotype in colon cancer. Mol Carcinog 48: 989-994, 2009

55. Landi S, Gemignani F, Canzian F, Gaborieau V, Barale R, Landi D, Szeszenia-Dabrowska N, Zaridze D, Lissowska J, Rudnai P, et al: DNA repair and cell cycle control genes and the risk of young-onset lung cancer. Cancer Res 66: 11062-11069, 2006.

56. Picelli S, Zajac P, Zhou XL, Edler D, Lenander C, Dalén J, Hjern F, Lundqvist N, Lindforss U, Påhlman L, et al: Common variants in human CRC genes as low-risk alleles. Eur J Cancer 46: 1041-1048, 2010.

57. Smith TR, Levine EA, Freimanis RI, Akman SA, Allen GO, Hoang KN, Liu-Mares W and Hu JJ: Polygenic model of DNA repair genetic polymorphisms in human breast cancer risk. Carcinogenesis 29: 2132-2138, 2008.

58. Li Z, Kong L, Yu L, Huang J, Wang K, Chen S, Yu M and Wei S: Association between MSH6 G39E polymorphism and cancer susceptibility: A meta-analysis of 7,046 cases and 34,554 controls. Tumour Biol 35: 6029-6037, 2014.

59. Chang YC, Chang JG, Liu TC, Lin CY, Yang SF, Ho CM, Chen WT and Chang YS: Mutation analysis of 13 driver genes of colorectal cancer-related pathways in Taiwanese patients. World J Gastroenterol 22: 2314-2325, 2016.
60. Kukita Y, Okami J, Yoneda-Kato N, Nakamae I, Kawabata T, Higashiyama M, Kato J, Kodama K and Kato K: Homozygous inactivation of CHEK2 is linked to a familial case of multiple primary lung cancer with accompanying cancers in other organs. Cold Spring Harb Mol Case Stud 2: a001032, 2016.

61. Talseth-Palmer BA, Bauer DC, Sjursen W, Evans TJ, McPhillips M, Proietto A, Otton G, Spigelman AD and Scott RJ: Targeted next-generation sequencing of 22 mismatch repair genes identifies Lynch syndrome families. Cancer Med 5: 929-941, 2016.

62. Lynch HT and Shaw TG: Practical genetics of colorectal cancer. Chin Clin Oncol 2: 12, 2013.

63. Warren JJ, Pohlhaus TJ, Changela A, Iyer RR, Modrich PL and Beese Lorena S: Structure of the Human MutSalpha DNA lesion recognition complex. Mol Cell 26: 579-592, 2007.

64. Clark AB, Deterding L, Tomer KB and Kunkel TA: Multiple functions for the N-terminal region of Msh6. Nucleic Acids Res 35: 4114-4123, 2007.

65. Derwahl M and Nicula D: Estrogen and its role in thyroid cancer. Endocr Relat Cancer 21: T273-T283, 2014.

66. Nielsen SM, White MG, Hong S, Aschebrook-Kilfoy B, Kaplan EL, Angelos P, Kulkarni SA, Olopade OI and Grogan RH: The breast-thyroid cancer link: A systematic review and meta-analysis. Cancer Epidemiol Biomarkers Prev 25: 231-238, 2016.

67. Chuffa LG, Lupi-Júnior LA, Costa AB, Amorim JP and Seiva FR: The role of sex hormones and steroid receptors on female reproductive cancers. Steroids 118: 93-108, 2017.

68. Cavalieri E and Rogan E: The molecular etiology and prevention of estrogen-initiated cancers: Ockham's Razor: Pluralitas non est ponenda sine necessitate. Plurality should not be posited without necessity. Mol Aspects Med 36: 1-55, 2014.

69. Yager JD: Mechanisms of estrogen carcinogenesis: The role of E2/E1-quinone metabolites suggests new approaches to preventive intervention-A review. Steroids 99: 56-60, 2015.

70. Vahteristo P, Ojala S, Tamminen A, Tommiska J, Sammalkorpi H, Kiuru-Kuhlefelt S, Eerola H, Aaltonen LA, Aittomäki K and Nevanlinna H: No MSH6 germline mutations in breast cancer families with colorectal and/or endometrial cancer. J Med Genet 42: e22, 2005.

71. Wada-Hiraike O, Yano T, Nei T, Matsumoto Y, Nagasaka K, Takizawa S, Oishi H, Arimoto T, Nakagawa S, Yasugi T, et al: The DNA mismatch repair gene $\mathrm{hMSH} 2$ is a potent coactivator of oestrogen receptor alpha. Br J Cancer 92: 2286-2291, 2005.

72. Nemec AA, Bush KB, Towle-Weicksel JB, Taylor BF, Schulz V, Weidhaas JB, Tuck DP and Sweasy JB: Estrogen drives cellular transformation and mutagenesis in cells expressing the breast cancer-associated R438W DNA polymerase lambda protein. Mol Cancer Res 14: 1068-1077, 2016.

73. Hsu HM, Wang HC, Chen ST, Hsu GC, Shen CY and Yu JC: Breast cancer risk is associated with the genes encoding the DNA double-strand break repair Mre11/Rad50/Nbs1 complex. Cancer Epidemiol Biomarkers Prev 16: 2024-2032, 2007.

74. Ming-Shiean H, Yu JC, Wang HW, Chen ST, Hsiung CN, Ding SL, Wu PE, Shen CY and Cheng CW: Synergistic effects of polymorphisms in DNA repair genes and endogenous estrogen exposure on female breast cancer risk. Ann Surg Oncol 17: 760-771, 2010.

75. Chang IY, Jin M, Yoon SP, Youn CK, Yoon Y, Moon SP Hyun JW, Jun JY and You HJ: Senescence-dependent MutS alpha dysfunction attenuates mismatch repair. Mol Cancer Res 6: 978-989, 2008 